\title{
Changes in particle transport as a result of resonant magnetic perturbations in DIII-D
}

S. Mordijck, E. J. Doyle, G. R. McKee, R. A. Moyer, T. L. Rhodes, L. Zeng, N. Commaux, M. E. Fenstermacher, K. W. Gentle, H. Reimerdes, O. Schmitz, W. M. Solomon, G. M. Staebler, and G. Wang

Citation: Physics of Plasmas 19, 056503 (2012); doi: 10.1063/1.4718316

View online: https://doi.org/10.1063/1.4718316

View Table of Contents: http://aip.scitation.org/toc/php/19/5

Published by the American Institute of Physics

\section{Articles you may be interested in}

Discovery of stationary operation of quiescent $\mathrm{H}$-mode plasmas with net-zero neutral beam injection torque and high energy confinement on DIII-D

Physics of Plasmas 23, 056103 (2016); 10.1063/1.4943521

The EPED pedestal model and edge localized mode-suppressed regimes: Studies of quiescent $\mathrm{H}$-mode and development of a model for edge localized mode suppression via resonant magnetic perturbations

Physics of Plasmas 19, 056115 (2012); 10.1063/1.3699623

Impurity confinement and transport in high confinement regimes without edge localized modes on DIII-D Physics of Plasmas 22, 055901 (2015); 10.1063/1.4918359

Plasma transport in stochastic magnetic field caused by vacuum resonant magnetic perturbations at diverted tokamak edge

Physics of Plasmas 17, 102503 (2010); 10.1063/1.3487733

Effect of island overlap on edge localized mode suppression by resonant magnetic perturbations in DIII-D Physics of Plasmas 15, 056122 (2008); 10.1063/1.2901064

Changes in density fluctuations as a result of resonant magnetic perturbations correlate with the density inverse scale length

Physics of Plasmas 19, 024504 (2012); 10.1063/1.3680641

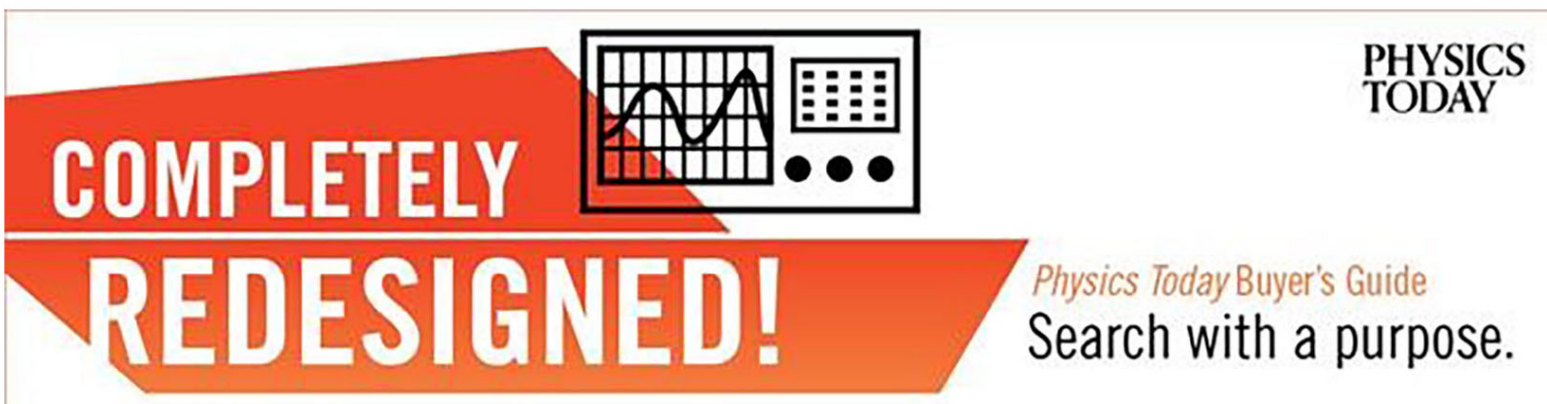




\title{
Changes in particle transport as a result of resonant magnetic perturbations in DIII-D ${ }^{a)}$
}

\author{
S. Mordijck, ${ }^{1, b)}$ E. J. Doyle, ${ }^{2}$ G. R. McKee, ${ }^{3}$ R. A. Moyer, ${ }^{4}$ T. L. Rhodes, ${ }^{2}$ L. Zeng, ${ }^{2}$ \\ N. Commaux, ${ }^{5}$ M. E. Fenstermacher, ${ }^{6}$ K. W. Gentle, ${ }^{7}$ H. Reimerdes, ${ }^{8}$ O. Schmitz, ${ }^{9}$ \\ W. M. Solomon, ${ }^{10}$ G. M. Staebler, ${ }^{11}$ and G. Wang ${ }^{2}$ \\ ${ }^{1}$ Department of Computer Science, College of William and Mary, Williamsburg, Virginia 23187, USA \\ ${ }^{2}$ Department of Physics and Astronomy, University of California - Los Angeles, Los Angeles, \\ California 90095, USA \\ ${ }^{3}$ Department of Engineering, University of Wisconsin, Madison, Wisconsin 53706, USA \\ ${ }^{4}$ Department of Mechanical and Aerospace Engineering, University of California, San Diego, La Jolla, \\ California 92093, USA \\ ${ }^{5}$ Oak Ridge National Laboratory, Oak Ridge, Tennessee 37831, USA \\ ${ }^{6}$ Lawrence Livermore National Laboratory, Livermore, California 94550, USA \\ ${ }^{7}$ Fusion Research Center, University of Texas at Austin, Austin, Texas 78712, USA \\ ${ }^{8}$ Columbia University, New York, New York 10027, USA \\ ${ }^{9}$ Institut für Energieforschung-Plasmaphysik, Forschungszentrum Jülich GmbH, Association EURATOM-FZJ \\ Trilateral Euregio Cluster, 52425 Juelich, Germany \\ ${ }^{10}$ Princeton Plasma Physics Laboratory, Princeton, New Jersey 08543, USA \\ ${ }^{11}$ General Atomics, P.O. Box 85608, San Diego, California 92186-5608, USA
}

(Received 21 December 2011; accepted 24 April 2012; published online 30 May 2012)

\begin{abstract}
In this paper, we introduce the first direct perturbed particle transport measurements in resonant magnetic perturbation (RMP) H-mode plasmas. The perturbed particle transport increases as a result of application of RMP deep into the core. In the core, a large reduction in $E \times B$ shear to a value below the linear growth rate, in conjunction with increasing density fluctuations, is consistent with an increase in turbulent particle transport. In the edge, the changes in turbulent particle transport are less obvious. There is a clear correlation between the linear growth rates and the density fluctuations measured at different scales, but it is uncertain which is the cause and which is the consequence. (C) 2012 American Institute of Physics. [http://dx.doi.org/10.1063/1.4718316]
\end{abstract}

\section{INTRODUCTION}

Resonant magnetic perturbations (RMPs) is the most promising technique that has been shown to suppress edge localized modes (ELMs) in three experimental devices (ASDEX-U, ${ }^{1}$ DIII-D, ${ }^{2}$ KSTAR (Ref. 3)) and mitigate them in additional devices (JET, ${ }^{4}$ MAST, ${ }^{5}$ NSTX (Ref. 6)). Discharges run in the ITER-like edge collisionality regime in DIII-D experience a large reduction in the density when the RMP is applied. The density reduction in the pedestal area is a large contributor to reducing the pressure gradient below the peeling-ballooning stability limit. In the core, however, the large reduction in density is a manifestation of a reduction in particle confinement, which can influence fusion performance in future tokamak devices, like ITER. In this paper, we will first show that RMP induced particle transport increases deep into the core of DIII-D high confinement H-mode plasmas. In the core, this increase can be related to a decrease of $E \times B$ shearing rate below the linear growth rate and increasing ionscale density fluctuations, which correlate with an increase in turbulent particle transport in this region. In the plasma edge, changes in linear growth rates correlate with changes in density fluctuations at ion-scale as well as intermediate scale.

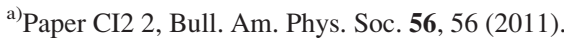

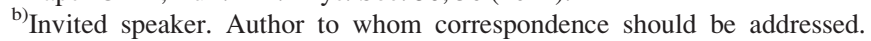
Electronic mail: mordijck@cs.wm.edu.
}

Previous work on particle transport and RMPs has mostly focused on changes in particle transport in the plasma edge. There is a large body of work on changes in stochastic magnetic diffusion, based on vacuum RMPs calculations. $^{7-10}$ This work has mostly focused on changes in stochastic magnetic diffusion in the plasma edge. Table I is a summary of this work for similar DIII-D H-mode plasmas, using quasi-linear approximations based on RechesterRosenbluth ${ }^{11}$ as well as those on field line tracing. In this table, $D_{s t}$ is the stochastic magnetic diffusion coefficient, $D_{M}=D_{s t} c_{s}$ is resulting particle transport, and $\chi_{e}=D_{s t} v_{e}$ is the resulting heat transport.

Using the same vacuum magnetic field modeling approach is the research on the creation of open field lines as a result of the vacuum magnetic fields. These open field lines act as an extended scrape-off layer, increasing parallel particle transport. ${ }^{12-14}$ Without reducing the heat flux to the divertor in simulations with ad-hoc flux limitations the reduction of the temperature profiles as a result of open field lines is too large in comparison with experiments. ${ }^{7,15,16}$ Another explanation for the changes in edge particle transport is based on changes in neoclassical transport. ${ }^{17,18}$ Neoclassical transport modeling shows that the radial electric field in the edge becomes less negative and closer to the ambipolar electric field, similarly to experimental results of the radial electric field. ${ }^{19}$ Finally, there are the changes in turbulence as a result of RMPs. The largest body of work on changes in turbulent 
TABLE I. Various results in the literature for stochastic magnetic diffusion.

\begin{tabular}{lcccc}
\hline \hline & Quasi-linear $^{7}$ & Quasi-linear $^{8}$ & Field line tracing & Field line tracing \\
& & \\
\hline & & $3.5 \times 10^{-6} \mathrm{~m}$ & $4.6 \times 10^{-7} \mathrm{~m}$ & \\
$D_{s t}$ & & $2 \mathrm{~m}^{2} / \mathrm{s}$ & $0.29 \mathrm{~m}^{2} / \mathrm{s}$ & \\
$D_{M}$ & $0.3-0.4 \mathrm{~m}^{2} / \mathrm{s}$ & $46 \mathrm{~m}^{2} / \mathrm{s}$ & $6.7 \mathrm{~m}^{2} / \mathrm{s}$ & $3 \mathrm{~m}^{2} / \mathrm{s}$ \\
$\chi_{e}$ & $20 \mathrm{~m}^{2} / \mathrm{s}$ & & \\
\hline \hline
\end{tabular}

particle flux as a result of RMPs was performed in low confinement L-mode. ${ }^{20,21}$ The only work on fluctuation changes as a result of RMPs in H-mode was performed on DIII-D. ${ }^{19,22,23}$ Moyer et al. ${ }^{22}$ showed at high collisionality that although magnetic fluctuations increase, there is no change to the radial electric field. However for low collisionality, the radial electric field in the pedestal area becomes more positive and at the same time the far infrared (FIR) coherent scattering (a non-localized measurement) shows that there is an increase in turbulence in the plasma edge. ${ }^{19}$ More recent work by $\mathrm{Yan}^{24}$ also shows a large increase in ion-scale density fluctuations deeper into the core, which respond instantly to changes in RMP amplitude. In this paper, we not only look at the changes in density fluctuations but also at the linear growth rates and $E \times B$ shear to understand what is causing these changes. In Sec. II, we will first introduce the two sets of experiments where the RMP amplitude was varied from discharge to discharge. Then in Sec. III, we introduce the first direct perturbative diffusion (D) and inward pinch (v) measurement changes as a result of RMPs. These indicate that particle transport increases over a large radial range, not just at the plasma edge as shown before. In Sec. IV, we look more specifically at changes in turbulent particle transport in the core. In Sec. V, the changes in fluctuations in the edge are compared to the changes in linear growth rates. Finally, we end with a discussion and conclusion.

\section{EXPERIMENTAL SETUP}

In this paper, we discuss two sets of discharges, where the RMP-coil current was varied from discharge to discharge. In this paper, we only discuss results for low collisionality discharges where $\nu^{*}=q_{95} R \epsilon^{-3 / 2} \lambda_{e}^{-1} \sim 0.05$. Here, $R$ is the major radius, $\epsilon(\equiv a / R)$ is the inverse aspect ratio, $a$ is the minor radius, $q_{95}$ is the safety factor at the $95 \%$ flux surface and $\lambda\left(=v_{T_{e}} \tau_{e}\right)$, the product of electron thermal velocity $v_{T_{e}}$, and collision time $\tau_{e}$ is the mean-free path for electron collisions. Both experimental setups are in the ITER similar shape (ISS), with average triangularity $\langle\delta\rangle \sim 0.53$. Both experimental setups are at similar conditions when RMP-coil $n=3$, with $n$ the toroidal model number, perturbation is applied; line averaged density $\left(5 \times 10^{19} \mathrm{~m}^{-3}\right), q_{95}(3.5$ versus 3.7$)$, plasma current $I_{p}=1.65 \mathrm{MA}$, axial toroidal magnetic field $B_{T}=1.9 \mathrm{~T}$.

Figures 1(a) and 1(b) show the time evolution of these discharges. Figure 1(a) illustrates that there is a reduction in the line-averaged density when we apply the RMP-coil current. If enough RMP-coil current is applied, then the ELMs are suppressed, as can be observed from the $D_{\alpha}$ trace. The discharges in second experiment [Fig. 1(b)] have a similar temporal evolution. The main differences are that the $q_{95}$ value is slightly lower but still inside the so-called resonant window for ELM suppression. The RMP-coil currents are slightly lower and suppression is observed at $4 \mathrm{kA}$. More importantly, in the second half of these discharges, starting at $2400 \mathrm{~ms}$ a modulated gas puff is applied. This gas puff causes the density to change and allows us to calculate the perturbed $D$ and $v$ from changes to the radial density profiles as measured by the reflectometer. ${ }^{25}$ For the discharges in Fig. 1(a), we compare the different density profiles at $3000 \mathrm{~ms}$, whereas for the discharges in Fig. 1(b), this analysis is performed at $2150 \mathrm{~ms}$ for the no RMP case and $2300 \mathrm{~ms}$ for the discharges with RMP-coil current. The modulated gas puff is applied later in the discharge and does not affect the reconstructed density profiles. For the discharges containing ELMs, we average over several $100 \mathrm{~ms}$ to obtain enough data points for the profile fit that are in the $80 \%-99 \%$ of the ELM cycle. For RMP ELM suppressed discharges $100 \mathrm{~ms}$ are enough to get sufficient data points for a good profile fit [Figs. 2(a) and 2(b)]. In

(a)

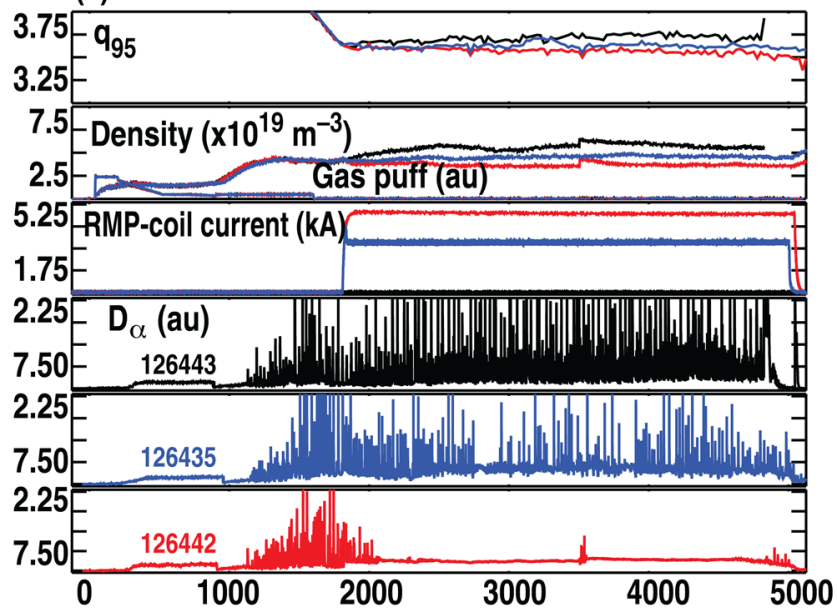

(b)

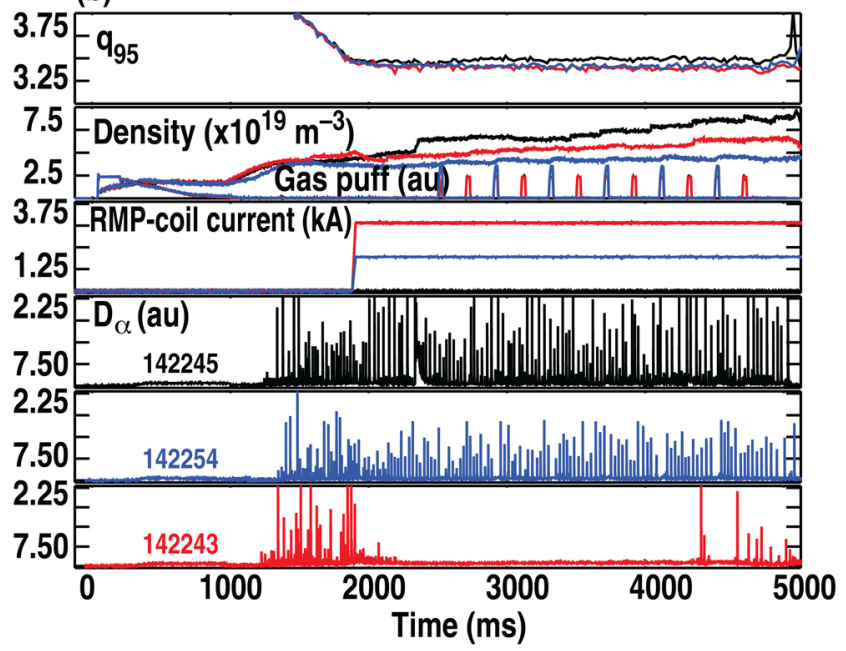

FIG. 1. Temporal evolution of the two experimental setups discussed in this paper. (a) In these discharges, $q_{95} \sim 3.5$ and the line integrated density before the RMP-coil is activated is $n_{e} \sim 5 \times 10^{19} \mathrm{~m}^{-3}$. During these discharges, a modulated gas puff is applied in order to obtain the perturbed $D$ and $v$ measurements. The RMP-coil current is varied from 0 to 4 and $6.2 \mathrm{kA}$ and at 6.2 kA ELMs are suppressed, as shown in the $D_{\alpha}$ traces. (b) These discharges are very similar to the ones in (a). The main difference is in a slightly higher $q_{95} \sim 3.7$ and lower RMP-coil currents, namely 2 and 4 kA. 

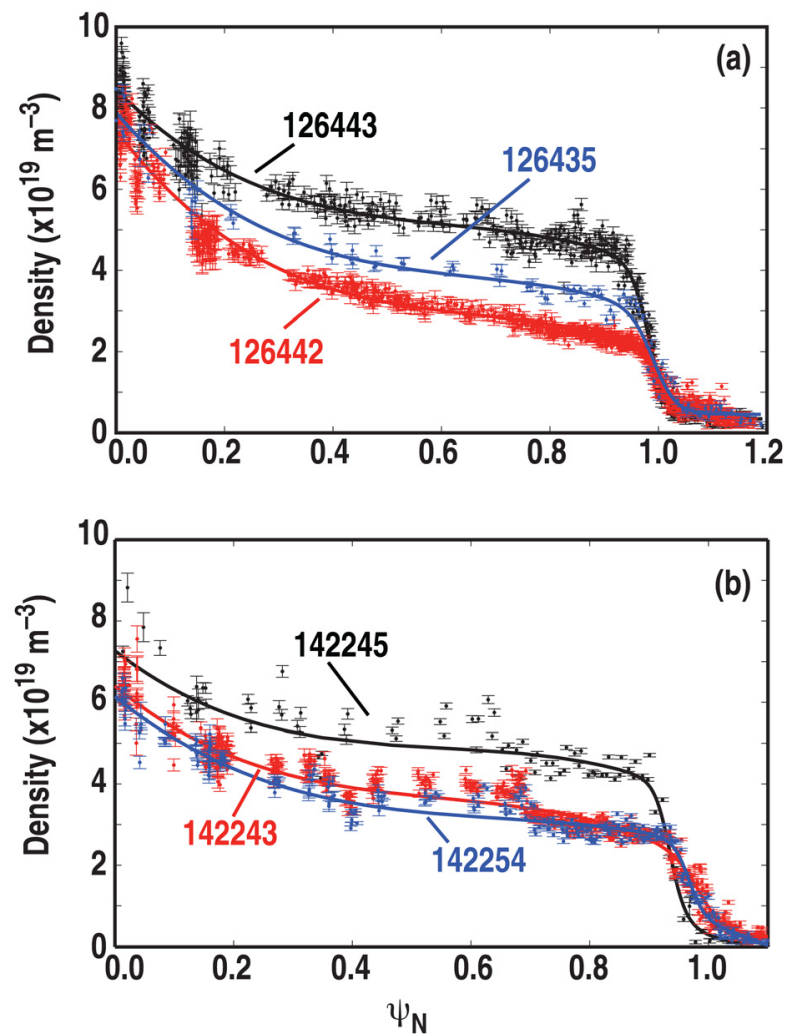

FIG. 2. Experimental density profiles in normalized poloidal flux $\Psi_{N}$ at $3000 \mathrm{~ms}$ from the Thomson scattering system with calculated fit for (a) discharges 126443 ( $0 \mathrm{kA}$ black), 126435 (4 kA blue), and 126442 (6.2 kA red); (b) discharges 142245 (0 kA black), 142254 (2 kA blue), and 142243 (4kA red). In all of these discharges, there is a reduction in the density profile that penetrates all the way into the core.

both figures, we observe that the changes to the density profile are not just limited to the pedestal area but that the density drops over the whole radial profile. In Sec. III, in order to characterize the changes in the density profile, we use a dimensionless number, the inverse density scale length, $a / L_{n}=a / n(d n / d r)$.

\section{CHANGES IN DENSITY PROFILES AND PARTICLE TRANSPORT}

Due to the fact that there is a global change in the density profiles [Figs. 2(a) and 2(b)], in order to be able to compare the changes in profile shape, we need to transform the profiles into a dimensionless number. The inverse density scale length $a / L_{n}$ captures the changes in profile shape while correcting for the global changes as a result of global density reduction. Figures 3(a) and 3(b) show the changes in $a / L_{n}$. Figure 3(a) focuses on changes to the core; here $a / L_{n}$ increases over the whole radius with increasing RMP-current, whereas in the pedestal area [Fig. 3(b)], $a / L_{n}$ decreases with increasing current. This illustrates that the changes to the density profile are not just limited to the plasma edge.

Similarly, the first perturbed $D$ and $v$ measurements show that the changes to the particle transport are not just limited to the plasma edge (Fig. 4). ${ }^{26}$ These $D$ and $v$ measurements are the result of the modulated gas puff experiments, which alter the density profiles.
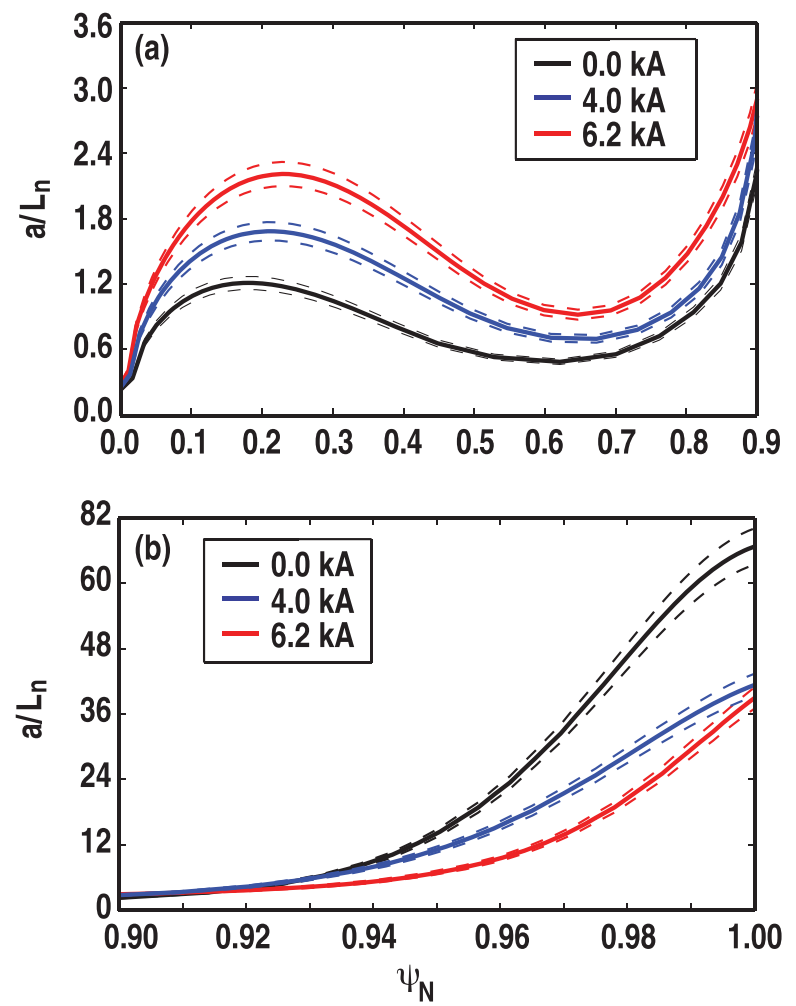

FIG. 3. $a / L_{n}$ with fitting uncertainties for three discharges: without RMP, $4 \mathrm{kA}$, and $6.2 \mathrm{kA}$. (a) shows the increase in $a / L_{n}$ from $\Psi_{N}=0-0.9$ with increasing RMP and (b) shows the decrease in $a / L_{n}$ from $\Psi_{N}=0.9-1$ with increasing RMP.

This is represented in the continuity equation by introducing a perturbed $\tilde{n}(r, t)=A(r) \exp (i[\omega t-\varphi(r)]$, see Eq. (1). Here $\tilde{n}$ is the perturbed density, $r$ is the radial location, $t$ is time, $A$ is the measured amplitude, $\omega$ is the measured frequency, and $\varphi$ is the measured phase shift of the perturbed density.

$$
\frac{\delta \tilde{n}}{\delta t}=-\frac{1}{r} \frac{\delta}{\delta r}\left(-r D \frac{\delta \tilde{n}}{\delta r}+r V \tilde{n}\right)+\tilde{S} .
$$

By rewriting the continuity equation in function of $D$ and $v$, together with the Fourier decomposition of the modulated

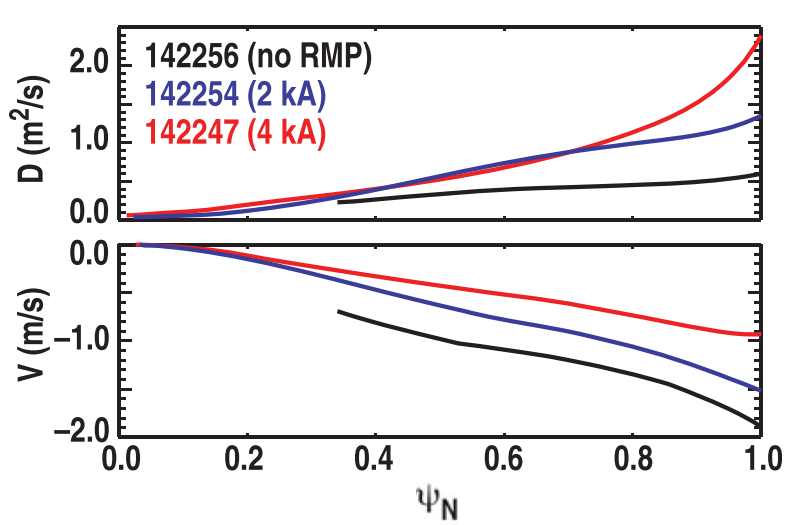

FIG. 4. Perturbed $D$ and $v$ measurements for three discharges with 0,2 , and $4 \mathrm{kA}$. $D$ increases with increasing RMP-coil current and the inward pinch $v$ decreases. 
density profiles (which give us $A$ and $\omega$ ), we can derive the perturbed diffusion and convection terms of the perturbative particle transport [Eqs. (2) and (3)]. Note that at this point, we are ignoring the perturbative particle source from the gas puffs, since no radial measurements of the fueling source are available for analysis. This technique is explained in more detail by Takenaga et al. $^{27}$

$$
\begin{gathered}
D=-\frac{\omega(Y \sin \varphi+X \cos \varphi)}{r(\delta \varphi / \delta r) A}, \\
V=-\frac{\omega(\delta A / \delta r Y-\delta \varphi / \delta r A X) \sin \varphi}{r(\delta \varphi / \delta r) A^{2}} \\
-\frac{\omega(\delta \varphi / \delta r A Y+\delta A / \delta r X) \cos \varphi}{r(\delta \varphi / \delta r) A^{2}},
\end{gathered}
$$

where $X=\int r A \cos \varphi d r$ and $Y=\int r A \sin \varphi d r$.

Increasing RMP-current reduces the perturbed inward pinch, $v$, and increases the perturbed diffusion, $D$ (see Figure 4). Both these results indicate that particle transport increases as a result of RMPs and that this increase is not just limited to the plasma edge. ${ }^{26}$ The results indicate that ratio of $v / D$ is reduced in magnitude when the RMP is applied over the whole radial region. The results based on the inverse density scale length indicate that there should be a difference between the edge of the plasma and the core, assuming that $\nabla n / n$ is proportional to $v / D$. The most logical explanation as to why the $v / D$ ratio in the edge does not follow the trend in $\nabla n / n$ is the result of the missing modulated neutral fueling source in our calculation of the perturbed D and $v$ measurements. In Sec. IV, we will look at the changes in turbulence characteristics in the core area and show that the changes are indicative of an increase in turbulent particle transport.

\section{CHANGES IN CORE TURBULENCE}

Generally, we observe an increase in density fluctuations when we apply $n=3$ RMPs in DIII-D H-mode plasmas. ${ }^{19,22,23}$ Figure 5 is an example of such an increase in density fluctua-

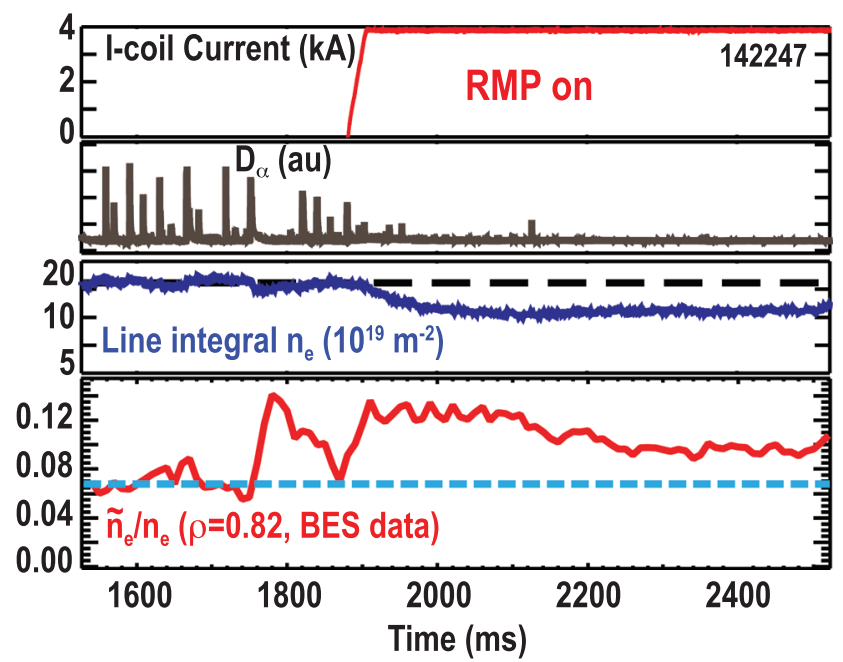

FIG. 5. Time evolution of a discharge with $4 \mathrm{kA}$ RMP-coil current. At $\rho \sim 0.82$, the density fluctuations at $k_{\theta} \rho_{s} \sim 0.3-0.5$ instantly increase when the coil current is applied. tions measured with the beam emission spectroscopy (BES). ${ }^{29}$ When the RMP-coil current is applied, the density pump-out is observed, but on a faster time-scale, there is an increase in the density fluctuations. The density fluctuations, $\tilde{n}$, at $k_{\theta} \rho_{s} \sim$ $0.3-0.5$ initially increase by $50 \%$, where $k_{\theta}$ is the wave number and $\rho_{s}$ is the ion sound gyroradius.

These increases are not only observed at normalize radius, $\rho \sim 0.82$ with the BES, but over a large radius from $\rho=0.7-0.85$ (Fig. 6). Figure 6 shows that the density fluctuations increase after the application of the RMP-coil current and that this increase is observed over a large radial core region. Previous work ${ }^{30}$ shows that there is no correlation between the changes in $a / L_{n}$ and the increase in $\tilde{n} / n$ in this region. However, a large reduction in the $E \times B$ shear is observed in this region. ${ }^{31}$ Figure 7 shows that the $E \times B$ shear is substantially reduced and that this reduction is the same for 4.0 and $6.2 \mathrm{kA}$ RMP-coil current in these discharges. By comparing the value of the $E \times B$ shearing rate with the linear growth rate, calculated by a trapped gyroLandau fluid transport model (TGLF), ${ }^{32}$ we observe that at $\Psi_{N} \sim 0.8$, the linear growth rate is larger than the $E \times B$ shearing rate for the RMP discharges $(E \times B \sim 50 \mathrm{krad} / \mathrm{s}$ versus $\gamma \sim 100 \mathrm{krad} / \mathrm{s})$. The linear growth rate at this radial location for $k_{\theta} \rho_{s} \sim 0.5$ remains similar for all the discharges, namely $100 \mathrm{krad} / \mathrm{s}$. All these changes indicate that turbulent particle transport increase in the core as a result of RMPs.

\section{CHANGES IN PEDESTAL TURBULENCE}

In the pedestal, the changes are more complex and a direct inference on changes to the particle transport are less obvious than in the core, where everything points to an increase in turbulent particle transport. Previous work did find a correlation between the changes in $a / L_{n}$ and $\tilde{n} / n$ at $k_{\theta} \rho_{s} \sim 0.5$, both values decrease with increasing RMP-coil current. $^{30}$ Based on these results, we calculated the linear growth with TGLF for $k_{\theta} \rho_{s} \sim 0.5$. The linear growth rate captures more information than the inverse density scale length. We find that at $\Psi_{N}=0.95$ the linear growth rate decreases, similarly as the $\tilde{n} / n$. In Fig. 8 , the $\tilde{n} / n$ are plotted

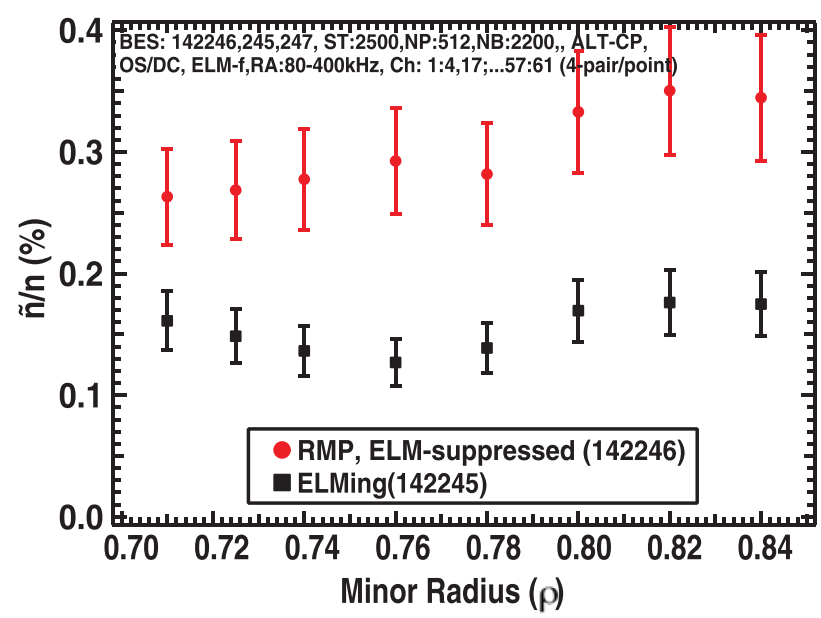

FIG. 6. Changes in $\tilde{n} / n$ as measured by the BES from $\rho=0.7$ up to $\rho=0.85$. Applying RMPs leads to an increase in $\tilde{n} / n$ over the whole measured radius. 


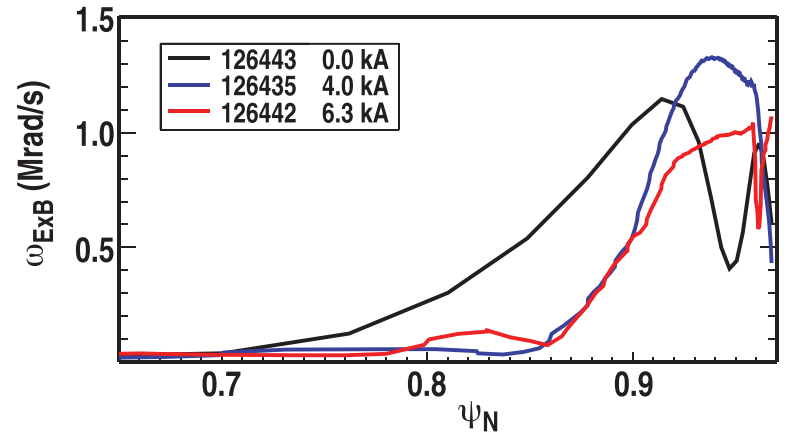

FIG. 7. $E \times B$ is strongly reduced as a result of RMPs from $\Psi_{N}=0.7$ up to $\Psi_{N}=0.9$.

versus $\gamma$ at $k_{\theta} \rho_{s} \sim 0.5$. Similarly to the results for $a / L_{n}$ versus $\tilde{n} / n$, we find a correlation.

Where previous results focused on ion-scale density fluctuations, in this paper we also present the density fluctuations of the Doppler backscattering (DBS) ${ }^{28}$ at medium-scale, $k_{\theta} \rho_{s}$ $\sim 1$ (Fig. 9). Where at ion-scale the density fluctuations decreased in the plasma edge, ${ }^{31}$ at medium scale they increase if enough RMP-coil current is employed (Fig. 9). If we compare the changes in linear growth rate at $k_{\theta} \rho_{s} \sim 1$ with $\tilde{n} / n$, although the behavior in $\tilde{n} / n$ at this scale is very different from that at ion-scale, there is again a correlation (Fig. 10). In this case, the linear growth rate actually increases, similarly to the density fluctuations.

In summary, in the pedestal, depending on what turbulence scale you observe, RMPs can increase or decrease density fluctuation levels. To complicate the relation to particle transport, there is no clear drop in the $E \times B$ shearing rate. ${ }^{31}$ This makes any interpretations as to the how turbulent particle transport changes in this area impossible.

\section{DISCUSSION AND CONCLUSIONS}

In the Sec. III, we introduced first measurements of the perturbed diffusion and inward pinch coefficients in RMP H-mode plasmas. These transport coefficients are not equal to the equilibrium transport. In order to connect the perturbed particle transport to the equilibrium transport, modeling of both the perturbed as well as the equilibrium conditions are needed to relate both to each other. Another

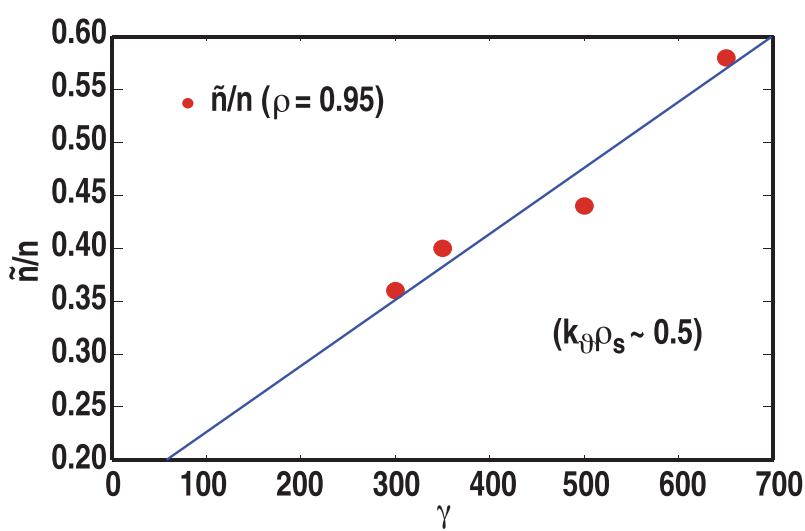

FIG. 8. Linear correlation between the linear growth rate calculated by TGLF and $\tilde{n} / n$ at $k_{\theta} \rho_{s} \sim 0.5$ from the BES.

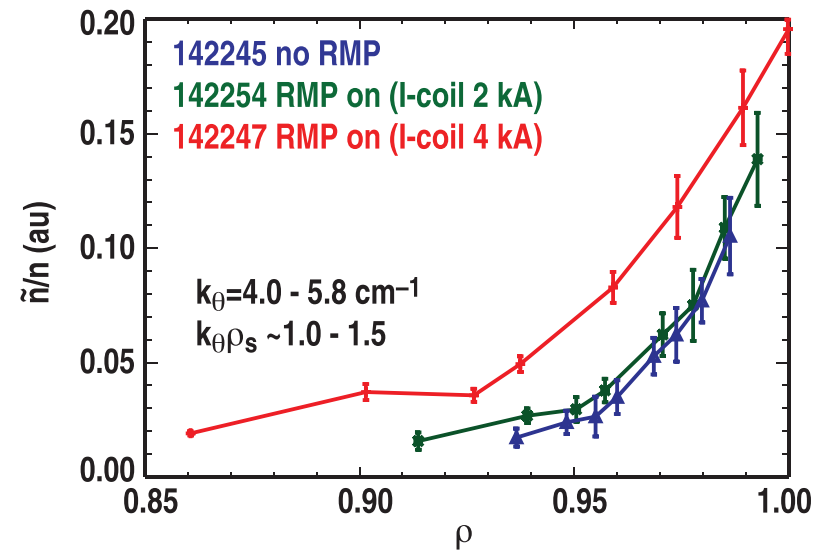

FIG. 9. Changes in $\tilde{n} / n$ at $k_{\theta} \rho_{s} \sim 1-1.5$ at the plasma edge. At this $k$-scale, $\tilde{n} / n$ increases only when enough ( $4 \mathrm{kA}$ in this case) RMP-coil current is applied.

aspect that needs to be addressed is the influence of the gas puff on the perturbed results themselves, especially at the plasma edge. Although the gas puff is only $10 \mathrm{~ms}$ long in duration, its magnitude is large enough to influence the perturbed measurements in the plasma edge. Therefore, future modeling of the particle transport with TGLF and the neutral fueling at the plasma edge are required to couple the perturbed particle transport to the equilibrium transport.

In Sec. IV, the large reduction in $E \times B$ shearing below the linear growth rate and an increase in $\tilde{n} / n$ are an indication that turbulent particle transport is increasing as a result of the applied RMPs. In order to make predictions for future machines, we need to understand what is causing this reduction in the $E \times B$ shearing rate. In Fig. 11, we show that there is a large change in the carbon toroidal rotation in this region of the plasma. The toroidal rotation decreases inside $\Psi \sim$ 0.85 and increases outside of this radius. This indicates a change in carbon toroidal rotational shear. This is the largest contributor to the changes in $E \times B$ shearing rate, since the changes in pressure gradient are mostly located in the pedestal area and not in the core.

The reduction in the $E \times B$ shearing rate is so substantial that understanding the changes in toroidal rotation are vital to predict the changes in particle transport and also the density pump-out. Previous work on non-resonant magnetic fields (NRMF) in QH-mode discusses the same

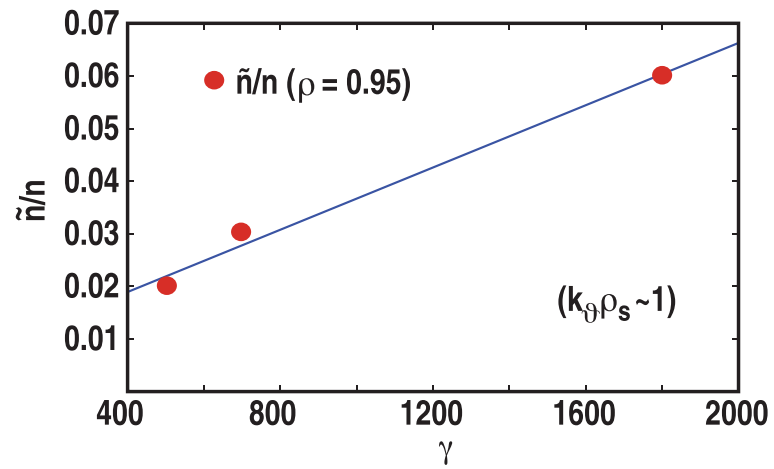

FIG. 10. Linear correlation between the linear growth rate calculated by TGLF and $\tilde{n} / n$ at $k_{\theta} \rho_{s} \sim 1$ from the DBS. 


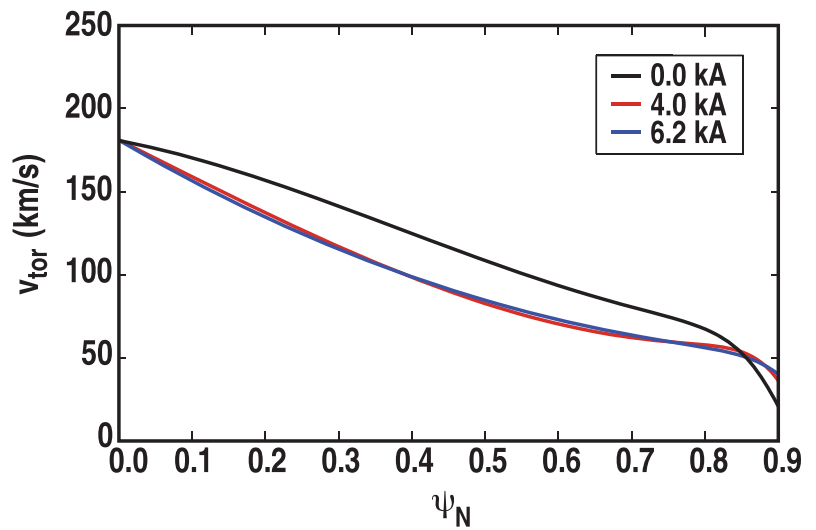

FIG. 11. Changes in the radial profile of the toroidal carbon rotation. The toroidal rotation decreases in the core and increases towards the edge.

phenomenon. ${ }^{33}$ In this paper, neoclassical toroidal viscosity (NTV) is playing a critical role in modifying the changes in toroidal rotation. Future modeling would be required to address the changes in toroidal rotation as a result of RMPs versus NRMFs.

Although the changes in turbulence characteristics in the pedestal area do not point to a clear change in particle transport, the fact that the linear growth rates correlate with the changes in density fluctuations at different k-scales is an important result (Sec. V). The main question in these results is, what is driving what. Are the changes in experimental profiles creating the changes in density fluctuations or are the changes in fluctuations leading to the changes in experimental profiles? In this paper, we only focus on the changes once the plasmas have reached a steady-state, in order to address this chicken and egg problem, detailed time-dependent analysis of the fluctuations and the growth rates is required. If this analysis would point out that the changes in turbulence are along for the ride in the pedestal area, there are still a vast number of theories (as discussed in the Introduction) that could explain the changes in particle transport in the pedestal.

One of the main concerns when explaining the changes in particle transport as a result of RMPs is the lack of changes in heat transport. Especially the changes in electron temperature appear non-existent at first glance. Where with particle transport, there is the global observation of the density pump-out and an overall reduction in density profiles, nothing similar exists for the heat transport. However, if you examine the changes in electron temperature more closely, by using a dimensionless number, $a / L_{T_{e}}=\left(a / T_{e}\right)\left(d T_{e} / d r\right)$, there are small but significant local changes in the electron temperature profile (Fig. 12). Future modeling of the heat transport is needed to address how much the heat transport changes.

To summarize, in this paper we introduced the first direct perturbed particle transport measurements in RMP $\mathrm{H}$-mode plasmas. The perturbed particle transport increases as result of RMP deep into the core. In the core, a large reduction in $E \times B$ shear, below the linear growth rate, in conjunction with increasing density fluctuations is consistent with an increase in turbulent particle transport. In the edge, the changes in turbulent particle transport are less clear. There is a clear correlation between the linear growth rates
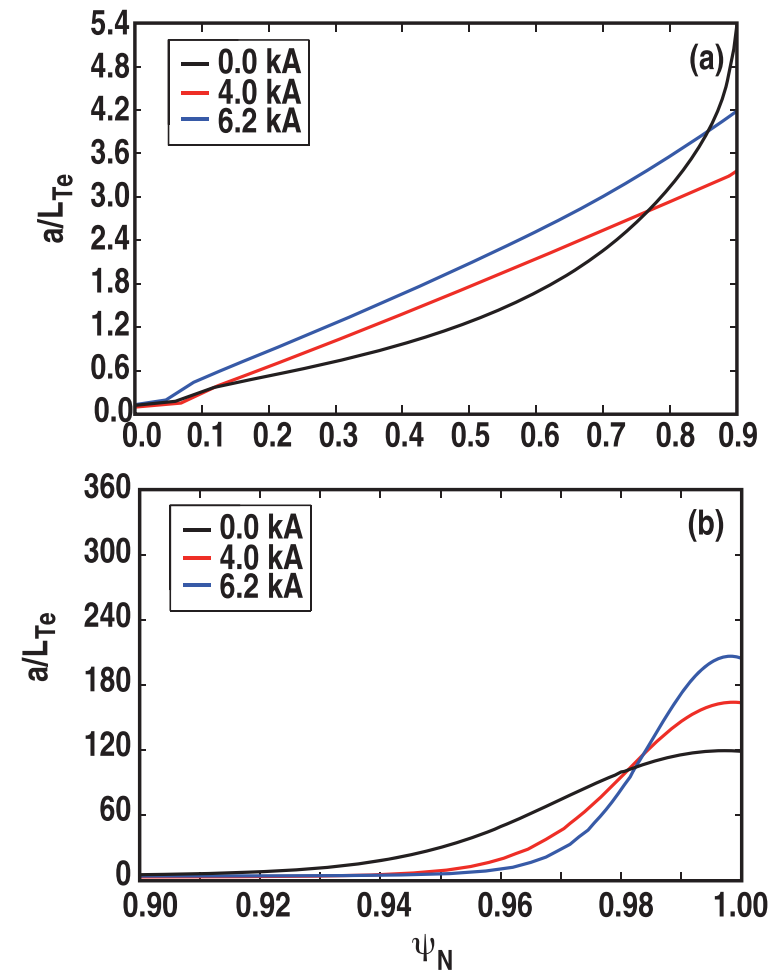

FIG. 12. $a / L_{T_{e}}$ for three discharges: without RMP, $4 \mathrm{kA}$, and $6.2 \mathrm{kA}$. (a) shows the increase in $a / L_{T_{e}}$ from $\Psi_{N}=0-0.9$ with increasing RMP and (b) shows the decrease in $a / L_{T_{e}}$ from $\Psi_{N}=0.9-1$ with increasing RMP.

and the density fluctuations measured at different scales, but it is unclear which is cause and which is consequence.

\section{ACKNOWLEDGMENTS}

This work was supported in part by the U.S. Department of Energy under DE-FG02-07ER54917, DE-FC0204ER54698, DE-FG02-89ER54139, DE-FG02-08ER54999, DE-AC05-00OR22725, DE-AC52-07NA27344, DE-FG0397ER54415, DE-FG02-04ER54761, DE-AC02-09CH11466, and DE-FG02-95ER54309. Special thanks to Pieter Peers.

${ }^{1}$ W. Suttrop, T. Eich, J. C. Fuchs, S. Günter, A. Janzer, A. Herrmann, A. Kallenbach, P. T. Lang, T. Lunt, M. Maraschek, R. M. McDermott, A. Mlynek, T. Pütterich, M. Rott, T. Vierle, E. Wolfrum, Q. Yu, I. Zammuto, and H. Zohm, Phys. Rev. Lett. 106, 225004 (2011).

${ }^{2}$ T. E. Evans, R. A. Moyer, P. R. Thomas, J. G. Watkins, T. H. Osborne, J. A. Boedo, E. J. Doyle, M. E. Fenstermacher, K. H. Finken, R. J. Groebner, M. Groth, J. H. Harris, R. J. La Haye, C. J. Lasnier, S. Masuzaki, N. Ohyabu, D. G. Pretty, T. L. Rhodes, H. Reimerdes, D. L. Rudakov, M. J. Schaffer, G. Wang, and L. Zeng, Phys. Rev. Lett. 92, 235003 (2004).

${ }^{3}$ E. Kwon, "KSTAR announces successful ELM suppression," ITER newsline (10 November 2011).

${ }^{4}$ Y. Liang, H. R. Koslowski, P. R. Thomas, E. Nardon, B. Alper, P. Andrew, Y. Andrew, G. Arnoux, Y. Baranov, M. Bécoulet, M. Beurskens, T. Biewer, M. Bigi, K. Crombe, E. De La Luna, P. de Vries, W. Fundamesnki, S. Gerasimov, C. Giroud, M. P. Gryaznevich, N. Hawkes, S. Hotchin, D. Howell, S. Jachmich, V. Kiptily, L. Moreira, V. Parail, S. D. Pinches, E. Rachlew, and O. Zimmermann, Phys. Rev. Lett. 98, 265004 (2007).

${ }^{5}$ A. Kirk, E. Nardon, R. Akers, M. Bécoulet, G. De Temmerman, B. Dudson, B. Hnat, Y. Q. Liu, R. Martin, P. Tamain, D. Taylor, and MAST Team, Nucl. Fusion 50, 034008 (2010).

${ }^{6}$ J. M. Canik, R. Maingi, T. E. Evans, R. E. Bell, S. P. Gerhardt, H. W. Kugel, B. P. LeBlanc, J. Manickam, J. E. Menard, T. H. Osborne, J.-K. Park, S. Paul, P. B. Snyder, S. A. Sabbagh, E. A. Unterberg, and NSTX Team, Nucl. Fusion 50, 034012 (2010). 
${ }^{7}$ I. Joseph, T. E. Evans, A. M. Runov, M. E. Fenstermacher, M. Groth, S. V. Kasilov, C. J. Lasnier, R. A. Moyer, G. D. Porter, M. J. Schaffer, R. Schneider, and J. G. Watkins, Nucl. Fusion 48, 045009 (2008).

${ }^{8}$ T. E. Evans, K. H. Burrell, M. E. Fenstermacher, R. A. Moyer, T. H. Osborne, M. J. Schaffer, W. P. West, L. W. Yan, J. A. Boedo, E. J. Doyle, G. J. Jackson, I. Joseph, C. J. Lasnier, A. W. Leonard, T. L. Rhodes, P. R. Thomas, J. G. Watkins, and L. Zeng, Phys. Plasmas 13, 056121 (2006).

${ }^{9}$ L. Yan and T. E. Evans, J. Nucl. Mater. 363-365, 723 (2007).

${ }^{10}$ W. M. Stacey and T. E. Evans, Phys. Plasmas 13, 112506 (2006).

${ }^{11}$ A. Rechester and M. Rosenbluth, Phys. Rev. Lett. 40, 38 (1978).

${ }^{12}$ M. W. Jakubowski, S. S. Abdullaev, K. H. Finken, M. Lehnen, and TEXTOR Team, J. Nucl. Mater. 337-339, 176 (2005).

${ }^{13}$ M. W. Jakubowski, M. Lehnen, K. H. Finken, O. Schmitz, S. S. Abdullaev, B. Unterberg, R. C. Wolf, and TEXTOR Team, Plasma Phys. Controlled Fusion 49, S109 (2007).

${ }^{14}$ O. Schmitz, M. W. Jakubowski, H. Frerichs, D. Harting, M. Lehnen, B. Unterberg, S. S. Abduallaev, S. Brezinsek, I. Classen, T. Evans, Y. Feng, K. H. Finken, M. Kantor, D. Riter, U. Samm, B. Schweer, G. Sergienko, G. W. Spakman, M. Tokar, E. Uzgel, R. C. Wolf, and TEXTOR Team, Nucl. Fusion 48, 024009 (2008).

${ }^{15}$ H. Frerichs, D. Reiter, O. Schmitz, T. E. Evans, and Y. Feng, Nucl. Fusion 50, 034004 (2010).

${ }^{16}$ O. Schmitz, T. E. Evans, M. E. Fenstermacher, H. Frerichs, M. W. Jakubowski, M. J. Schaffer, A. Wingen, W. P. West, N. H. Brooks, K. H. Burrell, J. S. deGrassie, Y. Feng, K. H. Finken, P. Gohil, M. Groth, I. Joseph, C. J. Lasnier, M. Lehnen, A. W. Leonard, S. Mordijck, R. A. Moyer, A. Nicolai, T. H. Osborne, D. Reiter, U. Samm, K. H. Spatschek, H. Stoschus, B. Unterberg, E. A. Unterberg, J. G. Watkins, R. Wolf, and DIII-D and TEXTOR Teams, Plasma Phys. Controlled Fusion 50, 124029 (2008).

${ }^{17}$ M. Z. Tokar, T. E. Evans, R. Singh, and B. Unterberg, Phys. Plasmas 15, 072515 (2008).

${ }^{18}$ G. Park, C. S. Chang, I. Joseph, and R. A. Moyer, Phys. Plasmas 17, 102503 (2010).

${ }^{19}$ R. A. Moyer, K. H. Burrell, T. E. Evans, M. E. Fenstermacher, P. Gohil, I. Joseph, T. H. Osborne, M. J. Schaffer, P. B. Snyder, J. G. Watkins, L. R. Baylor, M. Bécoulet, J. A. Boedo, N. H. Brooks, E. J. Doyle, K. H. Finken, P. Garbet, M. Groth, J. Harris, E. M. Hollmann, G. L. Jackson, M. Jakubowski, T. C. Jernigan, S. Kasilov, C. J. Lasnier, A. W. Leonard, M. Lehnen, J. Lonnroth, E. Nardon, V. Parail, G. D. Porter, T. Rhodes, D. L. Rudakov, A. Runov, O. Schmitz, R. Schneider, D. M. Thomas, P. Thomas, G. Wang, W. P. West, L. Yan, J. H. Yu, and L. Zeng, in Proceedings of the 21st IAEA Fusion Energy Conference, Chengdu, China (International Atomic Energy Agency, Vienna, 2006).

${ }^{20}$ Y. Xu, R. R. Weynants, S. Jachmich, M. Van Schoor, M. Vergote, P. Peleman, M. W. Jakubowski, M. Mitri, D. Reiser, B. Unterberg, K. H. Finken, and TEXTOR Team, Phys. Rev. Lett. 97, 165003 (2006).

${ }^{21}$ Y. Xu, M. Van Schoor, R. R. Weynants, S. Jachmich, M. Vergote, M. W. Jakubowski, P. Beyer, M. Mitri, B. Schweer, D. Reiser, B. Unterberg, K. H. Finken, M. Lehnen, R. Jaspers, and TEXTOR Team, Nucl. Fusion 47, 1696 (2007).

${ }^{22}$ R. A. Moyer, T. E. Evans, T. H. Osborne, P. R. Thomas, M. Becoulet, J. Harris, K.-H. Finken, J. A. Boedo, E. J. Doyle, M. E. Fenstermacher, P. Gohil, R. J. Groebner, M. Groth, G. L. Jackson, R. J. La Haye, C. J. Lasnier, A. W. Leonard, G. R. McKee, H. Reimerdes, T. L. Rhodes, D. L. Rudakov, M. J. Schaffer, P. B. Snyder, M. R. Wade, G. Wang, J. G. Watkins, W. P. West, and L. Zeng, Phys. Plasmas 12, 056119 (2005).

${ }^{23}$ S. Mordijck, "Particle transport as a result of resonant magnetic perturbations," Ph.D. dissertation (University of California, San Diego, 2011).

${ }^{24}$ Z. Yan, G. R. McKee, R. J. Groebner, P. B. Snyder, T. H. Osborne, M. N. A. Beurskens, K. H. Burrell, T. E. Evans, R. A. Moyer, H. Reimerdes, and $\mathrm{X} . \mathrm{Xu}$, in Proceedings of the 23rd IAEA Fusion Energy Conference, Daejeon, South Korea (International Atomic Energy, Vienna, 2010).

${ }^{25}$ L. Zeng, G. Wang, E. J. Doyle, T. L. Rhodes, W. A. Peebles, and Q. Peng, Nucl. Fusion 46, S677 (2006).

${ }^{26}$ S. Mordijck, L. W. Owen, and R. A. Moyer, Nucl. Fusion 50, 034006 (2010).

${ }^{27}$ H. Takenaga, K. Nagashima, A. Sakasai, T. Oikawa, and T. Fujita, Plasma Phys. Controlled Fusion 40, 183 (1998).

${ }^{28}$ J. C. Hillesheim, W. A. Peebles, T. L. Rhodes, L. Schmitz, T. A. Carter, P.-A. Gourdain, and G. Wang, Rev. Sci. Instrum. 80, 083507 (2009).

${ }^{29}$ G. R. McKee, R. J. Fonck, D. K. Gupta, D. J. Schlossberg, M. W. Shafer, R. L. Boivin, and W. M. Solomon, Plasma Fusion Res. 2, S1025 (2007).

${ }^{30}$ S. Mordijck, R. A. Moyer, and G. R. McKee, "Changes in density fluctuations as a result of magnetic perturbations correlate with the density inverse scale length," Phys. Plasmas 19, 024504 (2012).

${ }^{31}$ S. Mordijck, R. A. Moyer, A. Kirk, P. Tamain, D. Temple, G. R. McKee, and E. Nardon, Plasma Physics and Controlled Fusion 53, 122001 (2011).

${ }^{32}$ G. M. Staebler, J. E. Kinsey, and R. E. Waltz, Phys. Plasmas 14, 055909 (2007).

${ }^{33}$ A. M. Garofalo, W. M. Solomon, J.-K. Park, K. H. Burrell, J. C. DeBoo, M. J. Lanctot, G. R. McKee, H. Reimerdes, L. Schmitz, M. J. Schaffer, and P. B. Snyder, Nucl. Fusion 51, 083018 (2011). 